\title{
Photoswitchable Molecular Rings for Solar-Thermal Energy Storage
}

\author{
E. Durgun $*, \dagger, \ddagger, \S$ and Jeffrey C. Grossman $*, \dagger$
}

${ }^{\dagger}$ Department of Materials Science and Engineering, Massachusetts Institute of Technology, Cambridge, Massachusetts 02139, United States

${ }^{\ddagger}$ UNAM-National Nanotechnology Research Center and ${ }^{\S}$ Institute of Materials Science and Nanotechnology, Bilkent University, Ankara 06800, Turkey

\section{Supporting Information}

ABSTRACT: Solar-thermal fuels reversibly store solar energy in the chemical bonds of molecules by photoconversion, and can release this stored energy in the form of heat upon activation. Many conventional photoswichable molecules could be considered as solar thermal fuels, although they suffer from low energy density or short lifetime in the photoinduced high-energy metastable state, rendering their practical use unfeasible. We present a new approach to the design of chemistries for solar thermal fuel applications, wherein well-known photoswitchable molecules are connected by different linker agents to form molecular rings. This approach allows for a significant increase in both the amount of stored energy per molecule and the stability of the fuels. Our results suggest a range of possibilities for tuning the energy density and thermal stability as a function of the type of the photoswitchable molecule, the ring size, or the type of linkers.

SECTION: Energy Conversion and Storage; Energy and Charge Transport

$\mathrm{E}$ fficient utilization of the sun as a renewable and clean energy source is one of the greatest goals and challenges of this century due to the increasing demand for energy and its environmental impact. Numerous strategies exist to convert sunlight into useful forms of energy, including photocatalytic processes, artificial photosynthesis, ${ }^{1,2}$ photothermal power plants, ${ }^{3}$ and photovoltaic applications. ${ }^{4}$ An alternative strategy to these is to convert and store the sun's energy directly in the chemical bonds of metastable photoisomers of suitable molecular systems. The stored energy can then be released as heat on demand by an external trigger. Ideally both the photisomerization and heat release reactions reversibly occur in a closed-cycle without changing the chemical composition. ${ }^{5}$ The clear advantage of such an approach is that the same material both converts and stores the sun's energy, providing a rechargeable fuel that can be safely transported and used ondemand; the materials used could, in principle, be cheap, nontoxic and abundant, and the cycle can be repeated thousands of times without any emission or waste.

The idea of storing solar energy in chemical bonds was first introduced with the photoisomerization reactions of organic molecules (such as norbornadiene $\rightleftharpoons$ quadricyclane ${ }^{6,7}$ and the trans $\rightleftharpoons$ cis isomers of azobenzene ${ }^{8}$ ), although the concept was generally dismissed as unpractical ${ }^{7}$ due to a number of reasons including degradation, instability of the photoinduced isomer (limited half-life or shelf life), low energy density, and cost. As such, the majority of recent experimental studies for such materials have focused on their photoswitchable properties, ${ }^{9}$ as opposed to energy storage. The Ru-based organometallic complex (tetracarbonyl-diruthenium fulvalene) ${ }^{10}$ has shown promise as a solar thermal fuel due to its high cyclability without degradation; however, substitutions for $\mathrm{Ru}$ must be found to reduce costs, and the volumetric energy density increased for practical applications. ${ }^{11,12}$ Recently, a new strategy that combines photoswitchable molecules with nanoscale templates was shown to provide a promising platform for controlling both stability and energy density. ${ }^{13}$ In such materials, the template serves as a means to rigidly pack and order the photoswitch molecules such that chemical interactions between the molecules can be tuned independently for the ground and excited states.

In this work, we suggest a different approach to design materials for solar thermal fuel applications, wherein once again well-known photoswitchable molecules are constrained in such a way as to allow for the tailoring of both the energy stored and stability in the charged state. However, unlike the case of templates which introduce new chemistries, here we approach the problem by using strain, imposed by the formation of molecular rings, to engineer the properties of the photoisomers. Using azobenzene and stilbene as examples, we employ firstprinciples calculations to predict the energy stored $(\Delta H)$ and heat release reaction barriers $\left(E_{\mathrm{a}}\right)$ for a variety of such tailored ring systems. Our calculations reveal that, as in the case of packing photoswitches on nanoscale templates, functionalized ring systems provide a template for tuning the key properties of photoswitchable molecules, transforming them into competitive

Received: November 17, 2012

Accepted: February 20, 2013

Published: March 4, 2013 
solar-thermal fuels. In particular, our calculations show that through a combination of the ring size and linker chemistry the strain in these systems can be exploited to engineer both the energy density and stability.

The azobenzene molecule $(A B)^{14}$ can be photoisomerized reversibly from the more stable trans- to the cis-isomer. Because the process is fast (picoseconds) and has a high quantum yield, ${ }^{15} \mathrm{AB}$ has been considered for applications in optomechanical devices, ${ }^{16,17}$ biochemical applications, ${ }^{18}$ and photoaddressable storage media. ${ }^{19}$ The cis isomer is roughly $0.6 \mathrm{eV}$ higher in energy than the trans form. (See Figure 1.) As an

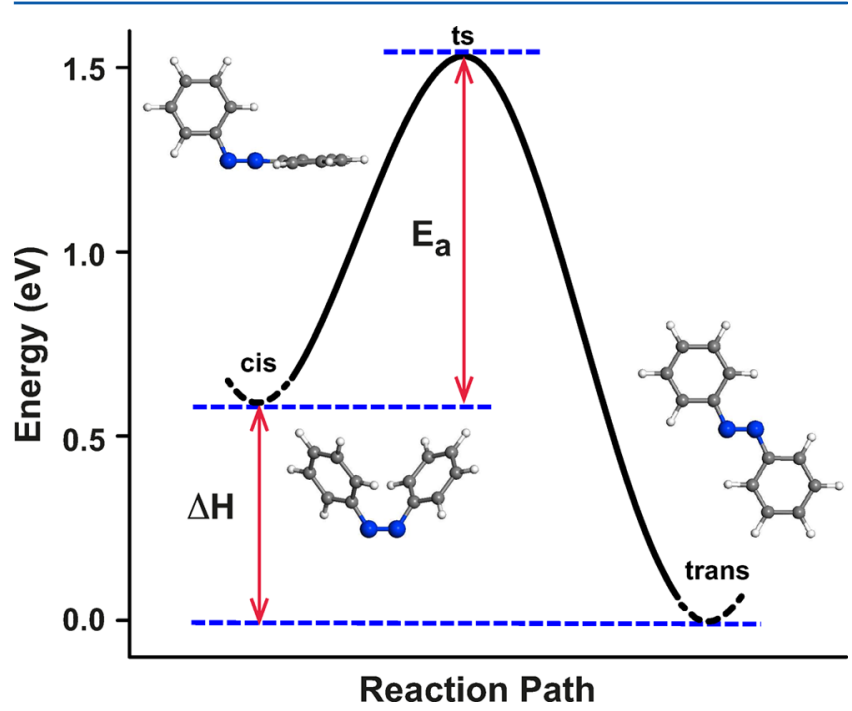

Figure 1. Back reaction (cis $\rightarrow$ trans) of a single azobenzene molecule in the gas phase. The cis, trans, and transition-state (ts) structures are shown where nitrogen, carbon, and hydrogen atoms are indicated by blue, gray, and white spheres, respectively.

energy storage medium ${ }^{20,8}$ this leads to a rather poor energy density, and, more importantly, the activation energy barrier of the cis $\rightarrow$ trans back reaction is low $(<1 \mathrm{eV})$, corresponding to a half life of only a few hours for the metastable state. ${ }^{20}$ No substitution pattern is known that significantly increases the activation barrier or the stored energy above that of unsubstituted $\mathrm{AB},{ }^{21}$ making it unpractical in its isolated state for solar thermal fuel applications.

Before considering ring systems, we first calculate $\Delta H$ and $E_{\mathrm{a}}$ for isolated $\mathrm{AB}$ (Figure 1). The cis isomer is found to be 0.58 $\mathrm{eV}$ higher in energy than the trans isomer, in good agreement with experimentally reported values. ${ }^{20,22}$ For the cis $\rightarrow$ trans thermal back reaction, ${ }^{23-25}$ we considered the inversion mechanism where one of the $\mathrm{N}$ atoms is inverted together with a perpendicular orientation of the $\mathrm{N}$-phenyl ring, as shown in Figure 1. Our computed $E_{\mathrm{a}}$ is $0.93 \mathrm{eV}$, which is also consistent with experimental data. ${ }^{20,26}$

For molecular azobenzene rings, or azobenzenophanes $[n-$ $\left(s_{1}, \ldots, s_{n}\right)$, where $n$ is the number of $\mathrm{AB}$ units and $s$ represents the cis (c) or trans (t) states], we first consider methylene $\left(\mathrm{CH}_{2}=\alpha\right)$ linkers and $n=2-6$. Azobenzenophanes with $\alpha$ linkers have been experimentally synthesized, ${ }^{27}$ and the effect of steric distortion on photoisomerization as well as the thermal stability have been studied for the case of $\mathrm{AB}$ dimers. ${ }^{28-30}$ Our results for the variation of $\Delta H$ with $n$ (for $n=2-6$ ) and structures for the 2-(t,t), 2-(c,c), 3- $(\mathrm{t}, \mathrm{t}, \mathrm{t}), 3-(\mathrm{c}, \mathrm{c}, \mathrm{c}), 4-(\mathrm{t}, \mathrm{t}, \mathrm{t}, \mathrm{t})$, and $4-(c, c, c, c)$ systems are shown in Figure $2 a$. For the dimer 2$(\mathrm{t}, \mathrm{t})$ the induced strain is so large that the trans units deform from their ideal planar geometry, resulting in a reversal of the trans/cis energy ordering for this smallest "ring" case. Recently, Norikane et al. ${ }^{30}$ synthesized stable 2-(c,c) and showed that the trans case exhibited thermal isomerization to the cis state as well as reversible photochemical isomerization, in agreement with our calculations.

As can be seen in Figure 2a, the case of the ring dimer is an exception, and for larger sizes all trans configurations are more stable than their cis counterparts. ${ }^{27}$ Whereas our computed $\Delta H$ for $3-\mathrm{AB}$ is $0.57 \mathrm{eV} / \mathrm{AB}$ (nearly identical to the isolated $\mathrm{AB}$ case), the energy stored per azobenzene molecule increases to $0.97 \mathrm{eV} / \mathrm{AB}$ for the four-member ring case, representing a $65 \%$ increase over that of the isolated $\mathrm{AB}$ molecule. This result demonstrates the potential for engineering properties of solar thermal fuels via curvature-induced strain. For this choice of photoswitch molecule and linker, the four-member ring induces more strain in the cis states compared with the trans states, leading to a substantial gain in energy storage. For larger rings, $\Delta H$ decreases as a function of size, gradually approaching the infinite ring limit of $0.61 \mathrm{eV} / \mathrm{AB}$, where the trans and cis units approach their isolated geometry. ${ }^{31}$

To understand further the trends of $\Delta H$ shown in Figure 2a, we calculated separately the energy variation of a single $A B$ molecule with respect to five different structural degrees of freedom, including torsion angles $(\angle 2112$ and $\angle 1123)$, bending angles $(\angle 112$ and $\angle 124)$, and linker bond angles $(\angle 454)$, as shown in Figure 3a. (The change in bond lengths is also taken into account, although they are found to be negligible.) Each of these energy components can be expressed as a function of the change in angle with respect to isolated $\mathrm{AB}$, and by calculating these energy components using the corresponding angles in the ring geometry the total energy of the $\mathrm{AB}$ unit in the ring systems can be estimated. As shown in Figure 3a, the variation in $\Delta H$ obtained with this method follows the same trend computed by $\mathrm{ab}$ initio calculations and indicates that the change in $\Delta H$ for the rings originates mainly from steric effects with respect to the isolated $\mathrm{AB}$ molecule imposed by $\alpha$ linkers and the ring framework. Both the cis and trans units (and the corresponding transition states) are destabilized with respect to their isolate counterparts by an amount of energy proportional to the induced strain. When this effect is larger for cis units than trans units, $\Delta H$ increases depending on the deviation from ideal geometry (Figure $3 \mathrm{~b}$ ), with a maximum when the strain of cis units is the largest while that of the trans units is the smallest. This type of simple fitting also allows us to predict the energy change in a wide range of possible ring geometries and sizes without carrying out further DFT calculations, but rather considering only the change in the five angles discussed above with respect to isolated $\mathrm{AB}$. For example one can estimate the maximum $\Delta H$ by calculating the maximum $\operatorname{strain}^{32-34}$ for which isolated cis- $\mathrm{AB}$ still remains stable, separately for all of the fitted structural degrees of freedom (Figure 3), and then using the corresponding angles in the model. Assuming a plausible ring geometry ${ }^{33}$ where all trans units are very close to their isolated counterpart but all cis units are under the calculated maximum strain, we predict that $\Delta H$ for such a hypothetical case could be as high as $2.5 \mathrm{eV} / \mathrm{AB}$.

In addition to the size of the ring, this kind of asymmetric strain engineering can also be controlled by the choice of linker chemistry. As an example, $\mathrm{N}_{2}(\beta)$ can also be used to connect the phenyl rings of isolated $A B$. For the case of $3-A B$, we compared the structures and $\Delta H$ trends of the rings formed with one, two, or all of the $\alpha$ linkers replaced by $\beta$ linkers [3- 
(a)
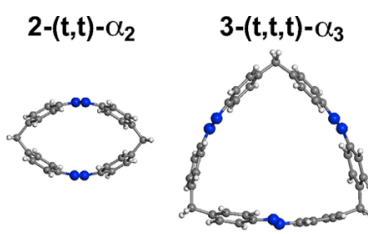

$2-(\mathrm{c}, \mathrm{c})-\alpha_{2}$

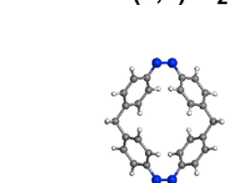

3-(c,c,c)- $\alpha_{3}$
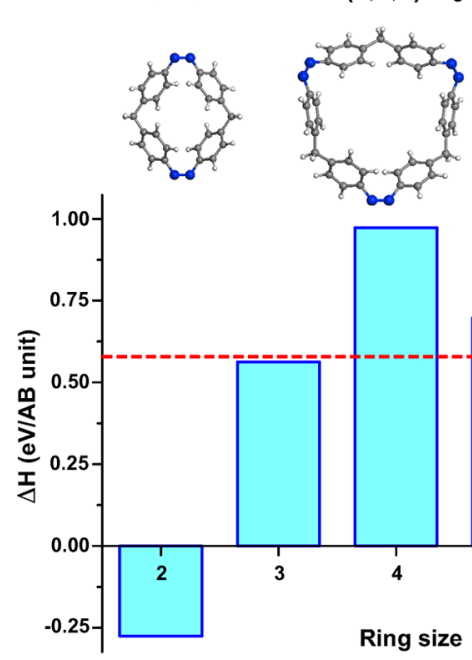

$4-(t, t, t, t)-\alpha_{4}$

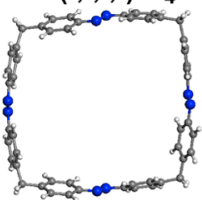

4-(c,c,c,c)- $\alpha_{4}$<smiles>[Mg][Mg]</smiles>

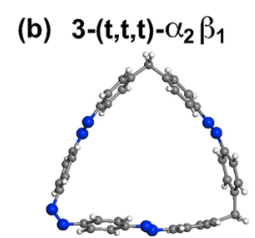

3-(c,c,c)- $\alpha_{2} \beta_{1}$
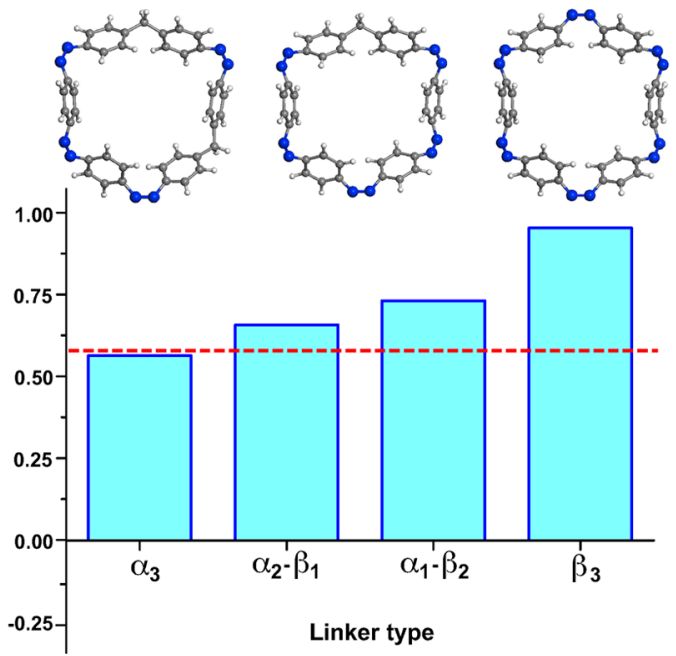

Figure 2. (a) Structure of 2-(t,t)- $\alpha_{2}, 2-(\mathrm{c}, \mathrm{c})-\alpha_{2}, 3-(\mathrm{t}, \mathrm{t}, \mathrm{t})-\alpha_{3}, 3-(\mathrm{c}, \mathrm{c}, \mathrm{c})-\alpha_{3}, 4-(\mathrm{t}, \mathrm{t}, \mathrm{t}, \mathrm{t})-\alpha_{4}$, and 4-(c,c,c,c)- $\alpha_{4}$ and the variation of $\Delta H$ with number of $\mathrm{AB}$ units. (b) Structure of 3-(t,t,t)- $\alpha_{2} \beta_{1}, 3-(\mathrm{c}, \mathrm{c}, \mathrm{c})-\alpha_{2} \beta_{1}, 3-(\mathrm{t}, \mathrm{t}, \mathrm{t})-\alpha_{1} \beta_{2}, 3-(\mathrm{c}, \mathrm{c}, \mathrm{c})-\alpha_{1} \beta_{2}$, and 3-(t,t,t)- $\beta_{3}, 3-(\mathrm{c}, \mathrm{c}, \mathrm{c})-\beta_{3}$ and the variation of $\Delta H$ with type of linker. The nitrogen, carbon, and hydrogen atoms are indicated by blue, gray, and white spheres, respectively.
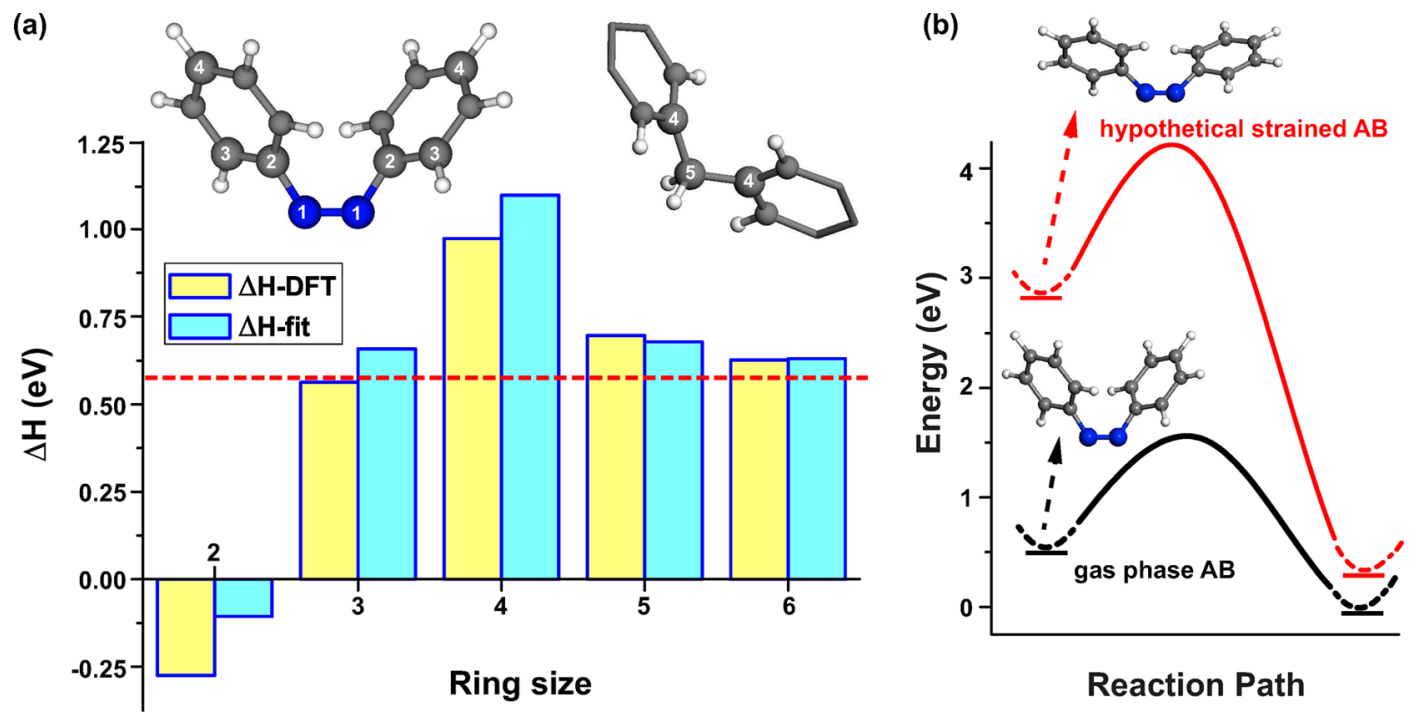

Figure 3. (a) Comparison of $\Delta H$ computed by DFT calculations and calculated by an energy decomposition methods. (See the text.) The atoms are labeled in order to define the five energy components including torsion, bending, and linker angles. (See the text.) $\Delta H$ for an isolated $\mathrm{AB}$ molecule is indicated by the red dashed line. (b) Energy variation of cis, trans, and transition states with and without strain is shown by black and red lines, respectively.

$\mathrm{AB}-\alpha_{3}, 3-\mathrm{AB}-\alpha_{2} \beta_{1}, 3-\mathrm{AB}-\alpha_{1} \beta_{2}$ and $\left.3-\mathrm{AB}-\beta_{3}\right]$ (Figure 2b). Interestingly $\Delta H$ increases for each additional $\beta$ and reaches up to $0.95 \mathrm{eV} / \mathrm{AB}$ for $3-\mathrm{AB}-\beta_{3}$, as shown in Figure $2 \mathrm{~b}$, considerably larger than the $0.57 \mathrm{eV} / \mathrm{AB}$ for the 3-AB- $\left(\alpha_{3}\right)$ case. For the $\beta$-linker, which forms the same nitrogen double bond as in $\mathrm{AB}, \Delta H$ increase because of the new bond formation and the linker bonding angle (between the linker and phenyl rings). Similar to isolated $\mathrm{AB}$ when the linker bonding angle (thus, the geometry) is close to the $\angle 112$ of trans $A B$, the system becomes energetically more favorable. When all $A B$ units are connected with $\beta$ linkers, $\mathrm{AB}$ molecules become indistinguishable, and the system turns into a ring of phenyl rings connected by $\mathrm{N}_{2}$. For this case, the ground-state configuration is obtained when $3-(t, t, t)-\beta_{3}$ is in a circular shape, as illustrated in Figure $2 \mathrm{~b}$, which is unique to $3-(\mathrm{t}, \mathrm{t}, \mathrm{t})-\beta_{3}$, where there are no more $\alpha$ linkers attempting to preserve the tetrahedral angle between $\mathrm{C}-\mathrm{C}-\mathrm{C}(4-5-4)$ bonds, thus inducing strain on the system.

When the ring size is larger than $3-\mathrm{AB}, \beta$ linkers start to destabilize the cis- $\mathrm{AB}$ units they are connecting by converting them into trans- $\mathrm{AB}$ upon geometry relaxation according to our 
calculations. As an example, for 4-AB- $\alpha_{3} \beta_{1} \Delta H$, where all $\mathrm{AB}$ units are still in the cis form, $\Delta H$ increases to $0.65 \mathrm{eV} / \mathrm{AB}$ compared with the isolated case, but it decreases upon additional $\beta$ linkers as cis- $\mathrm{AB}$ units start to transform into the trans form. Finally, when all $\alpha$ linkers are replaced by $\beta$, all cis$\mathrm{AB}$ units are converted into trans- $\mathrm{AB}$, making 4-(c,c,c,c)- $\beta_{4}$ unstable. (See the Supporting Information.) Similar to 3- $(\mathrm{t}, \mathrm{t}, \mathrm{t})$ $\beta_{3}, 4-(\mathrm{t}, \mathrm{t}, \mathrm{t}, \mathrm{t})-\beta_{4}$ also has a circular shape, and even if the initial configuration is $4-(c, c, c, c)-\beta_{4}$ the circular geometry with all trans units is the final structure after optimization.

In addition to ring size and linker chemistry, another degree of freedom for modifying the energy storage can be introduced by adding hydrogen bonds that vary in number between the cis and trans states, similar to what was recently demonstrated in templated chromophore systems. ${ }^{13}$ Toward this end, we consider a redesigned ring structure with a single $\mathrm{AB}$ with two $\mathrm{OH}$ groups, as shown in Figure 4. The addition of hydroxyl

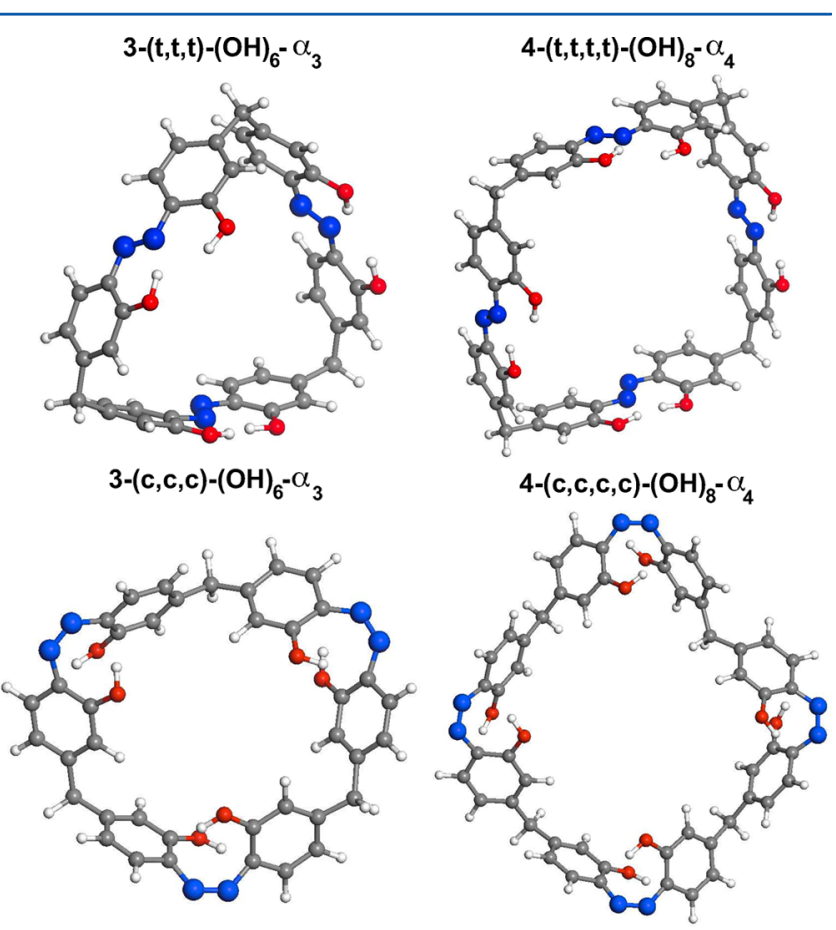

Figure 4. Structures of OH-substituted 3-(c,c,c), 3-(t,t,t), 4-(c,c,c,c), and $4-(t, t, t, t)$ structures.

groups in this way leads to an increases in $\Delta H$ to $0.99 \mathrm{eV} / \mathrm{AB}$ and $1.20 \mathrm{eV} / \mathrm{AB}$ for $3-\mathrm{AB}$ and $4-\mathrm{AB}$, respectively, corresponding to a 68 and $103 \%$ increase when compared with isolated $\mathrm{AB}$. Whereas strain induced by the ring framework destabilizes the two isomers (vide supra), the new H-bond formation stabilizes them with different amounts of energy. Our results indicate that these two mechanisms can be combined and the relative difference in the stabilizing and destabilizing energy of cis and trans units can provide even larger values of $\Delta H$.

Considering the ring structure as a torus and assuming maximum packing efficiency, the maximum volumetric and gravimetric energy densities of our calculated azobenzenophanes would be $1.2 \mathrm{MJ} / \mathrm{L}$ and $0.60 \mathrm{MJ} / \mathrm{kg}$, which are significantly larger than solid azobenzene $(\sim 0.32 \mathrm{MJ} / \mathrm{L}, \sim 0.32$ $\mathrm{MJ} / \mathrm{kg}$ ). These values could be even larger considering the predicted hypothetical $\Delta H$ value from our strain analysis model.
As discussed, concomitant with higher storage densities, the ability to control the stability of a solar thermal fuel in its charged state is of paramount importance to its practical use. Toward this end, we have computed the back reaction barrier $\left(E_{\mathrm{a}}\right)$ and possible reaction paths for all cis-azobenzenophanes. For example, for the case of $4-\mathrm{AB}$, we consider three different reaction paths: I: $(\mathrm{c}, \mathrm{c}, \mathrm{c}, \mathrm{c}) \rightarrow(\mathrm{t}, \mathrm{c}, \mathrm{c}, \mathrm{c}) \rightarrow(\mathrm{t}, \mathrm{t}, \mathrm{c}, \mathrm{c}) \rightarrow(\mathrm{t}, \mathrm{t}, \mathrm{t}, \mathrm{c}) \rightarrow$ $(\mathrm{t}, \mathrm{t}, \mathrm{t}, \mathrm{t})$, II: $\quad(\mathrm{c}, \mathrm{c}, \mathrm{c}, \mathrm{c}) \rightarrow(\mathrm{t}, \mathrm{t}, \mathrm{c}, \mathrm{c}) \rightarrow(\mathrm{t}, \mathrm{t}, \mathrm{t}, \mathrm{c}) \rightarrow(\mathrm{t}, \mathrm{t}, \mathrm{t}, \mathrm{t})$, and III: $(\mathrm{c}, \mathrm{c}, \mathrm{c}, \mathrm{c}) \rightarrow(\mathrm{t}, \mathrm{t}, \mathrm{t}, \mathrm{t}) . E_{\mathrm{a}}$ is calculated as $1.16,2.71$, and $3.17 \mathrm{eV}$ for paths I, II, and III, respectively, which suggests that the heat release reaction for such ring systems will be stepwise as opposed to concerted (see Figure 5) in agreement with

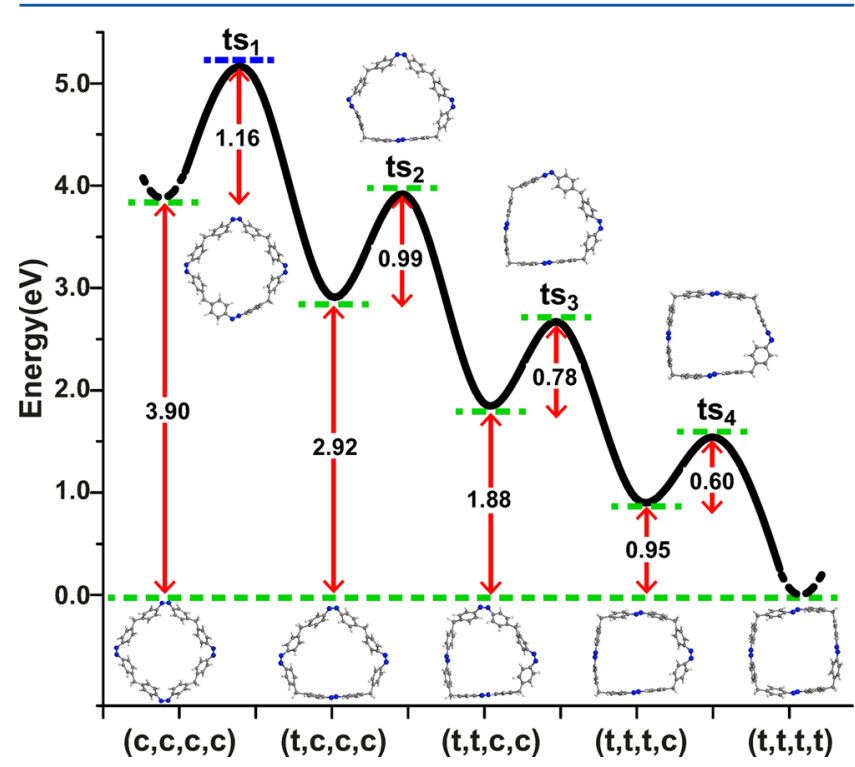

Figure 5. Computed stepwise back reaction path from the 4-(c,c,c,c)$\alpha_{4}$ to $4-(\mathrm{t}, \mathrm{t}, \mathrm{t}, \mathrm{t})-\alpha_{4}$ isomer of azobenzenophane. $E_{\mathrm{a}}$ and $\Delta H$ values and corresponding structures for each step are shown.

experimental data. ${ }^{27}$ For path I, $E_{\mathrm{a}}$ is largest for the first step and decreases for subsequent steps, indicating that the stability of $4-(c, c, c, c)$ is mainly determined by the first cis $\rightarrow$ trans barrier. We carried out similar sets of nudged-elastic band calculations (see Supporting Information) to predict $E_{\mathrm{a}}$ for the 3-AB, 5-AB, and $6-A B$ ring systems as well as for $3-A B$ with $\beta$ linkers, summarized in Table 1 . When compared with isolated $A B$, the ring geometry shows an increase in $E_{\mathrm{a}}$ and follows the same qualitative trend as $\Delta H$ with ring size. The energy decomposition model discussed above is also able to capture both the magnitude and mechanism of the variation of $E_{a}$ computed by ab initio calculations. As an example, an examination of the structural degrees of freedom in the model reveals that during the back reaction of $4-(c, c, c, c)$, when one cis unit transforms into the transition state $\left(\mathrm{ts}_{1}\right)$ as shown in Figure 5, the ring framework not only induces strain on the converted unit but also induces additional strain on the other three cis units, which results in an increase in the total energy of $\mathrm{ts}_{1}$ and thus in $E_{\mathrm{a}}$. For the second $E_{\mathrm{a}}\left(\mathrm{ts}_{2}\right)$, because one cis-unit is already converted to the trans-state, the additional strain reduces and $E_{\mathrm{a}}$ decreases, and so on. Accordingly, we can conclude that the induced strain not only increases the total energy of all-cis- $\mathrm{AB}$ configurations but also stabilizes the photoinduced high-energy metastable state. From the computed $E_{\mathrm{a}}$ values, the half life of the cis states can be predicted, ${ }^{36}$ and considering an average increase of $20 \%$ for $E_{a}$ compared 
Table 1. Variation of First Activation Energy $\left(E_{\mathrm{a}}\right)$ with ring size $(n=2-6)$ and Type of Linkers for 3-AB ${ }^{a}$

\begin{tabular}{|c|c|c|c|c|c|c|c|c|}
\hline ring size $(n)$ & 1 & & & & & 4 & 5 & 6 \\
\hline linker type & & $\alpha_{3}$ & $\alpha_{2} \beta_{1}$ & $\alpha_{1} \beta_{2}$ & $\beta_{3}$ & $\alpha_{4}$ & $\alpha_{5}$ & $\alpha_{6}$ \\
\hline$E_{\mathrm{a}}(\mathrm{eV})$ & 0.93 & 0.98 & 1.13 & 1.35 & 1.25 & 1.16 & 1.11 & 1.03 \\
\hline
\end{tabular}

with isolated $\mathrm{AB}$, the metastable cis-azobenzenophanes would have a half-life of greater than 1 year. ${ }^{37}$ For the system under maximum strain discussed above, although it is challenging to determine the exact corresponding reaction path, we computed $E_{\mathrm{a}}$ for a number of different possible transition states and found a range of 0.9 to $1.1 \mathrm{eV}$, indicating that the activation energy for this hypothetical, extreme case would lie in the same range as our reported values for the other ring systems. A similar trend and mechanism are observed for $3-\mathrm{AB}$ rings with $\beta$ linkers, where the addition of each $\beta$-linker uniformly increases $E_{\mathrm{a}}$ of the first step, except for $3-\mathrm{AB}-\beta_{3}$, in which case all $\mathrm{AB}$ units become indistinguishable, as discussed above.

In addition to thermodynamic considerations, the effect of the ring geometry or induced strain on the absorption spectrum is another important issue. It is well known that the absorption spectrum of single trans- $\mathrm{AB}$ shows a weak band at $450 \mathrm{~nm}$ $\left(n-\pi^{*}\right.$ excitation) and a strong one at $320 \mathrm{~nm}\left(\pi-\pi^{*}\right) .^{36}$ Norikane et al. ${ }^{27}$ synthesized and studied the absorption spectra of similar ring structures (two- to four-member rings with methylene linkers) and reported that the absorption maxima of trans isomers slightly shifted when compared with $\mathrm{AB}$ (by a maximum $10 \mathrm{~nm}$ to shorter or longer wavelengths for different sizes) but still remained near $320 \mathrm{~nm}$. According to our calculations, when compared with isolated trans- $\mathrm{AB}$, the energy gap of trans-azobenzenophanes is slightly reduced due to the induced strain, resulting in a small shift of the absorption maxima toward longer wavelengths. Thus, large changes in the absorption spectra are not expected for the ring structures considered here, in good agreement with previous experimental measurements. Systems with different linkers or two-member rings that are under very high strain could certainly have bigger changes in their absorption features. ${ }^{38,39}$

We believe the trends observed for ring systems presented here are not just limited to azobenzene but rather applicable to a wide range of photoconvertible molecules. To test one additional case, we analyze the stilbene molecule (SB), which is a prototype molecule for investigating photoisomerization effects and applications and has been the subject of a substantial number of experimental and theoretical studies. ${ }^{40}$ For isolated $\mathrm{SB}$, our calculated $\Delta H$ is $0.22 \mathrm{eV}$, in agreement with experimental measurements that report a value of $0.20 \mathrm{eV}$ in benzene solution. ${ }^{41}$ Similar to the $\mathrm{AB}$ case, for 3-SB $\Delta H$ slightly decreases to $0.19 \mathrm{eV} / \mathrm{SB}$ but significantly increases to $0.65 \mathrm{eV}$ for 4-SB, and using $\beta$-linkers instead of $\alpha$ also increases $\Delta H$ up to $0.68 \mathrm{eV}$ for $4-\mathrm{SB}$. Isolated cis-SB is rather unstable, with an experimentally measured $E_{\mathrm{a}}$ for $\mathrm{c} \rightarrow \mathrm{t}$ isomerization of $\sim 0.05$ $\mathrm{eV}^{42,43}$ and our computed $E_{\mathrm{a}}$ for isolated $\mathrm{SB}$ is $0.06 \mathrm{eV}$, consistent with the reported values. However, when in a ring form, just as for the azobenzenophanes, our calculations predict that for stilbenophanes $E_{\mathrm{a}}$ significantly increases up to 0.38 and $0.44 \mathrm{eV}$ for 3-SB-(c,c,c)- $\alpha_{3}$ and 3-SB-(c,c,c)- $\beta_{3}$, respectively.

In conclusion, we have shown that the molecular rings formed by connecting well-known photoswitchable molecules by suitable linker agents can simultaneously increase the stored energy and the reaction barrier of the back reaction of the photoswitch. These results indicate that the formation of rings of photoswitches holds the potential to be a promising template concept for solar-thermal fuels, with a number of degrees of freedom including the ring size, type of linker, H-bonding, or type photoconvertible molecule.

\section{METHODOLOGY}

Our calculations were performed within density functional theory (DFT) ${ }^{44}$ using PAW potentials ${ }^{45}$ implemented in the VASP package. ${ }^{46}$ The exchange-correlation potential was approximated within the generalized gradient approximation (GGA), ${ }^{47}$ and to test the functional dependence of our results we also carried out calculations using the HSE06 hybrid functional. ${ }^{48,49}$ The Brillouin zone integration was performed at the gamma point. We used a plane-wave basis set with a kinetic energy cutoff of $500 \mathrm{eV}$, which was checked to be sufficient to obtain fully converged results. All structures were considered as isolated molecules in a vacuum and were relaxed using the Kosugi algorithm (special Davidson block iteration scheme) with simultaneous minimization of the total energy and interatomic forces. The convergence on the total energy was set to $10^{-5} \mathrm{eV}$, and the maximum residual force allowed on each atom was fixed at $10^{-2} \mathrm{eV} / \AA$. For each ring system, a number of simulations was carried out from multiple different initial configurations and relaxed without any constraints to obtain ground-state structures. Minimum energy path calculations were performed using the nudged-elastic band approach. $^{50}$

\section{ASSOCIATED CONTENT}

\section{Supporting Information}

Variation of $\Delta H$ with different types of linkers for $4-\mathrm{AB}$ and computed stepwise back reaction path from the $3-(\mathrm{c}, \mathrm{c}, \mathrm{c})-\alpha_{3}$ to $3-(t, t, t)-\alpha_{3}$. This material is available free of charge via the Internet at http://pubs.acs.org.

\section{AUTHOR INFORMATION}

\section{Corresponding Author}

*E-mail: durgun@unam.bilkent.edu.tr (E.D.); jcg@mit.edu (J.C.G.).

\section{Notes}

The authors declare no competing financial interest.

\section{ACKNOWLEDGMENTS}

We are grateful for financial support from ARPA-E under contract number DE-AR0000180. Calculations were performed in part at the National Energy Research Scientific Computing Center, which is supported by the Office of Science of the U.S. Department of Energy under contract no. DE-AC0205CH11231 and in part by the National Science Foundation through TeraGrid resources provided by TACC under grant number TG-DMR090027. We are grateful to Dr. Alexie Kolpak for insightful discussions. 


\section{REFERENCES}

(1) Special Issue: Solar Fuels via Artificial Photosynthesis. Acc. Chem. Res. 2009, 42, 1859-2029.

(2) Lewis, N. S.; Nocera, D. G. Powering the Planet: Chemical Challenges in Solar Energy Utilization. Proc. Natl. Acad. Sci. 2006, 103, 15729-15735.

(3) Kenisarin, M.; Mahkamov, K. Solar Energy Storage Using Phase Change Materials. Renew. Sust. Energ. Rev. 2007, 11, 1913-1965.

(4) Nozik, A. J.; Miller, J. Introduction to Solar Photon Conversion. Chem. Rev. 2010, 110, 6443-6445.

(5) Kucharski, T. J.; Tian, Y.; Akbulatov, S.; Boulatov, R. Chemical Solutions for the Closed-Cycle Storage of Solar Energy. Energy Environ. Sci. 2011, 4, 4449-4472.

(6) Zou, J.-J.; Liu, Y.; Pan, L.; Wang, L.; Zhang, X. Photocatalytic Isomerization of Norbornadiene to Quadricyclane Over Metal (V, Fe and Cr)-incorporated Ti-MCM-41. Appl. Catal., B 2010, 95, 439-445.

(7) Philippopoulos, C.; Economou, D.; Economou, C.; Marangozis, J. Norbornadiene-quadricyclane System in the Photochemical Conversion and Storage of Solar Energy. Ind. Eng. Chem. Prod. Res. Dev. 1983, 22, 627-633.

(8) Olmsted, J., III; Lawrence, J.; Yee, G. G. Photochemical Storage Potential of Azobenzenes. Sol. Energy 1983, 30, 271-274.

(9) Kay, E. R.; Leigh, D. A.; Zerbetto, F. Synthetic Molecular Motors and Mechanical Machines. Angew. Chem., Int. Ed. 2007, 46, 72-191.

(10) Boese, R.; Cammack, J. K.; Matzger, A. J.; Pflug, K.; Tolman, W. B.; Vollhardt, K. P. C.; Weidman, T. W. Photochemistry of (Fulvalene)tetracarbonyldiruthenium and Its Derivatives: Efficient Light Energy Storage Devices. J. Am. Chem. Soc. 1997, 119, 67576773.

(11) Kanai, Y.; Srinivasan, V.; Meier, S. K.; Vollhardt, K. P. C.; Grossman, J. C. Mechanism of Thermal Reversal of the (Fulvalene)tetracarbonyldiruthenium Photoisomerization: Toward Molecular Solar-Thermal Energy Storage. Angew. Chem. Int. Edit 2010, 49, 8926-8929.

(12) Moth-Poulsen, K.; Coso, D.; Borjesson, K.; Vinokurov, N.; Meier, S. K.; Majumdar, A.; Vollhardt, K. P. C.; Segalman, R. A. Molecular solar thermal (MOST) energy storage and release system. Energy Environ. Sci. 2012, 5, 8534-8537.

(13) Kolpak, A. M.; Grossman, J. C. Azobenzene-Functionalized Carbon Nanotubes As High-Energy Density Solar Thermal Fuels. Nano Lett. 2011, 11, 3156-3162.

(14) Natansohn, A.; Rochon, P. Photoinduced Motions in AzoContaining Polymers. Chem. Rev. 2002, 102, 4139-4176.

(15) Granucci, G.; Persico, M. Excited State Dynamics with the Direct Trajectory Surface Hopping Method: Azobenzene and Its Derivatives as a Case Study. Theor. Chem. Acc. 2007, 117, 1131-1143.

(16) Browne, W. R.; Feringa, B. L. Making Molecular Machines Work. Nat. Nanotechnol. 2002, 1, 25-35.

(17) Hugel, T.; Holland, N. B.; Cattani, A.; Moroder, L.; Seitz, M.; Gaub, H. E. Single-Molecule Optomechanical Cycle. Science 2002, 296, 1103-1106.

(18) Khan, A.; Kaiser, C.; Hecht, S. Prototyp eines Photoschaltbaren Foldamers. Angew. Chem. 2006, 118, 1912-1915.

(19) Yu, Y.; Nakano, M.; Ikeda, T. Directed Bending of a Polymer Film by Light. Nature 2003, 425, 145.

(20) Taoda, H.; Hayakawa, K.; Kawase, K.; Yamakita, H. Photochemical Conversion And Storage Of Solar Energy By Azobenzene. J. Chem. Eng. Jpn. 1987, 20, 265.

(21) Tsuji, T.; Ohkita, M.; Kawai, H. Preparation and Kinetic Stabilization of Highly Strained Paracyclophanes. Bull. Chem. Soc. Jpn. 2002, 75, 415-433.

(22) Adamson, A. W.; Vogler, A.; Kunkely, H.; Wachter, R. Photocalorimetry. Enthalpies of Photolysis of Trans-Azobenzene, Ferrioxalate and Cobaltioxalate Ions, Chromium Hexacarbonyl, and Dirhenium Decarbonyl. J. Am. Chem. Soc. 1978, 100, 1298-1300.

(23) Tiberio, G.; Muccioli, L.; Berardi, R.; Zannoni, C. How Does the Trans-Cis Photoisomerization of Azobenzene Take Place in Organic Solvents? ChemPhysChem. 2010, 11, 1018-1028.
(24) Cimiraglia, R.; Hofmann, H.-J. Rotation and Inversion States in Thermal E/Z Isomerization of Aromatic Azo Compounds. Chem. Phys. Lett. 1994, 217, 430-435.

(25) Cattaneo, P.; Persico, M. An Ab initio Study of the Photochemistry of Azobenzene. Phys. Chem. Chem. Phys. 1999, 1, 4739-4743.

(26) Brown, E. V.; Granneman, G. R. Cis-Trans Isomerism in the Pyridyl Analogs of Azobenzene. Kinetic and molecular orbital analysis. J. Am. Chem. Soc. 1975, 97, 621-627.

(27) Norikane, Y.; Kitamoto, K.; Tamaoki, N. Novel Crystal Structure, Cis-Trans Isomerization, and Host Property of MetaSubstituted Macrocyclic Azobenzenes with the Shortest Linkers. J. Org. Chem. 2003, 68, 8291-8304.

(28) Rau, H.; Lueddecke, E. On the Rotation-Inversion Controversy on Photoisomerization of Azobenzenes. Experimental Proof of Inversion. J. Am. Chem. Soc. 1982, 104, 1616-1620.

(29) Nagamani, S. A.; Norikane, Y.; Tamaoki, N. Photoinduced Hinge-Like Molecular Motion: Studies on Xanthene-Based Cyclic Azobenzene Dimers. J. Org. Chem. 2005, 70, 9304-9313.

(30) Norikane, Y.; Katoh, R.; Tamaoki, N. Unconventional Thermodynamically Stable Cis Isomer and Trans to Cis Thermal Isomerization in Reversibly Photoresponsive [0.0](3,3')-Azobenzenophane. Chem. Commun. 2008, 1898-1900.

(31) $\Delta H$ for the infinite ring is $30 \mathrm{meV} / \mathrm{AB}$ larger than isolated $\mathrm{AB}$ due to the bonding between the $\alpha$-linker and phenyl rings.

(32) The maximum angle or torsional strain on isolated azobenzene for each angular component is determined when $\mathrm{d}^{2} E T / \mathrm{d} \theta$ is zero, where $E T$ is the total energy of single azobenzene molecule and $\theta$ is one of the bonding, bending, or torsional angles defined in the text.

(33) Senger, R. T.; Tongay, S.; Dag, S.; Durgun, E.; Ciraci, S. Carbon String Structures: First principles Calculations of Quantum Conductance. Phys. Rev. B 2005, 71, 235406.

(34) Durgun, E.; Senger, R. T.; Sevinçli, H.; Mehrez, H.; Ciraci, S. Spintronic Properties of Carbon-Based One-Dimensional Molecular Structures. Phys. Rev. B 2006, 74, 235413.

(35) It is hard to predict the corresponding ring geometry, but instead we design isolated cis- and trans-AB units by considering all of the corresponding angles and calculated the total energies without relaxations. The calculated $\Delta H$ is $>2 \mathrm{eV}$, indicating the possibility of the predicted value.

(36) Uchida, K. Photochromism. Molecules and Systems. Edited by Heinz Durr and Henri Bouas-Laurent. Angew. Chem., Int. Ed. 2004, 43, 3362.

(37) This corresponds to a thermal reversion (cis $\rightarrow$ trans) rate of $<0.1 \%$ per day.

(38) Bassotti, E.; Carbone, P.; Credi, A.; Di Stefano, M.; Masiero, S.; Negri, F.; Orlandi, G.; Spada, G. P. Effect of Strain on the Photoisomerization and Stability of a Congested Azobenzenophane: A Combined Experimental and Computational Study. J. Phys. Chem. A 2006, 110, 12385-12394.

(39) Hirose, Y.; Yui, H.; Sawada, T. Effect of Potential Energy Gap between the n-pi* and the pipi* State on Ultrafast Photoisomerization Dynamics of an Azobenzene Derivative. J. Phys. Chem. A 2002, 106, 3067-3071.

(40) Waldeck, D. H. Photoisomerization Dynamics of Stilbenes. Chem. Rev. 1991, 91, 415-436.

(41) Saltiel, J.; Ganapathy, S.; Werking, C. The $\Delta H$ for Thermal trans/cis-Stilbene Isomerization: Do $\mathrm{S}_{0}$ and $\mathrm{T}_{1}$ Potential Energy Curves Cross? J. Phys. Chem. 1987, 91, 2755-2758.

(42) Abrash, S.; Repinec, S.; Hochstrasser, R. M. The Viscosity Dependence and Reaction Coordinate for Isomerization of cisStilbene. J. Chem. Phys. 1990, 93, 1041-1053.

(43) Todd, D. C.; Jean, J. M.; Rosenthal, S. J.; Ruggiero, A. J.; Yang, D.; Fleming, G. R. Fluorescence Upconversion Study of cis-Stilbene Isomerization. J. Chem. Phys. 1990, 93, 8658-8668.

(44) (a) Kohn, W.; Sham, L. J. Self-Consistent Equations Including Exchange and Correlation Effects. Phys. Rev. 1965, 140, A1133A1138. (b) Hohenberg, P.; Kohn, W. Inhomogeneous Electron Gas. Phys. Rev. 1964, 136, B864-B871. 
(45) Blöchl, P. E. Projector Augmented-Wave Method. Phys. Rev. B 1994, 50, 17953-17979.

(46) (a) Kresse, G.; Hafner, J. Ab initio Molecular Dynamics for Liquid Metals. Phys. Rev. B 1993, 47, 558-561. (b) Kresse, G.;

Furthmuller, J. Efficient Iterative Schemes for $A b$ initio Total-Energy Calculations Using a Plane-Wave Basis Set. Phys. Rev. B 1996, 54, 11169-11186

(47) Perdew, J. P.; Burke, K.; Ernzerhof, M. Generalized Gradient Approximation Made Simple. Phys. Rev. Lett. 1996, 77, 3865-3868.

(48) Paier, J.; Hirschl, R.; Marsman, M.; Kresse, G. The PerdewBurke-Ernzerhof Exchange-Correlation Functional Applied to the G2-1 Test Set Using a Plane-Wave Basis Set. J. Chem. Phys. 2005, $122,234102$.

(49) Heyd, J.; Scuseria, G. E.; Ernzerhof, M. Erratum: "Hybrid Functionals Based on a Screened Coulomb Potential” [J. Chem. Phys. 118, 8207 (2003)]. J. Chem. Phys. 2006, 124, 219906.

(50) Mills, G.; Jonsson, H.; Schenter, G. K. Reversible Work Transition State theory: Application to Dissociative Adsorption of Hydrogen. Surf. Sci. 1995, 324, 305-337. 